\title{
Le ultime Cortes di Castiglia
}

\section{The last Cortes of Castile}

\author{
Fernando DE ANGELIS \\ Investigador \\ Dipartimento di Economia e Impresa \\ Università degl Studi della Tuscia di Viterbo \\ fernando_de_angelis@tiscali.it
}

Recibido: 12 de enero de 2015

Aceptado: 30 de marzo de 2015

\section{RESUMEN}

El artículo trata de examinar algunos aspectos relevantes (representación, poder, competencias, capacidades, etc.) de las últimas Cortes del Antiguo Régimen celebradas en España, las Cortes de Madrid del año 1789, cuando en Francia comenzaban a ponerse los cimientos del nuevo Régimen Liberal, lo que muestra la ambivalencia del momento y el retardo de nuestra práctica política para seguir los derroteros europeos: al absolutismo borbónico cerraba el paso a los aires revolucionarios franceses, pero la proximidad geográfica y cultural no podía impedir el efecto contagio. Sin embargo, esos nuevos aires no aflorarán hasta transcurridos casi veinte años, con el proceso que se inicia en 1808.

PALABRAS CLAVE: Ilustración, Antiguo Régimen, Revolución Francesa, Cortes, Representación, Constitución Histórica.

\begin{abstract}
The article intends to focus some relevant aspects (representation, power, competitions, capacities, etc.) of the last Ancien Regime Cortes celebrated in Spain, the 1789 Madrid Cortes, while in France the foundations of the new Liberal Regime were already starting. This Cortes clearly show the ambivalence of the moment and the delay of our political practice to follow the European courses: the Bourbon absolutism was blocking the way to the revolutionary French trends, while the geographical and cultural proximity could not prevent the contagion effect. Neverthless, these new trens will not come to light after almost twenty years later, with the process that begins in 1808 .
\end{abstract}

KEYWORDS: Illustration, Ancien Regime, French Revolution, Cortes (Spanish Parliament), Representation, Historical Constitution.

\section{RÉSUMÉ}

Le présent étude essaie d'examiner quelques aspects éminents (représentation, pouvoir, concurrences, capacités, etc.) des derniers Cortes de l'Ancien Régime célébrées en Espagne, les Cortes de Madrid reunies en 1789, quand en France oncommençait déjà à mettre en oeuvre les fondations du nouveau Régime Libéral, ce qui montre l'ambivalence du moment et le retard de notre pratique politique pour suivre les routes européennes. L'absolutisme bourbonnien fermait le chemin aux airs révolutionnaires français, mais la proximité géographique et culturelle ne pouvait pas empêcher l'effet de contagion. Cependant, ces nouveaux airs ne se feront présents après vingt ans écoulés, avec le processus qui s'initie en 1808 .

MOTS CLÉ : Lumières, Ancien Régime, Révolution Française, Cortes, Représentation, Constitution Historique. 
SUMARIO: Premessa. Paragrafo Primo: Brevi note sulle Cortes di antico regime. Paragrafo Due: le ultime Cortes di Antico Regime. Una breve conclusione.

\section{Premessa}

Con queste pagine ci si propone di delineare alcuni importanti aspetti degli ultimi scorci dell'Antico Regime in una terra, la Spagna del 1789, ancora lontana dai venti rivoluzionari francesi, ma, allo stesso tempo, vicina culturalmente al Paese dei Lumi.

Proprio in quell'anno si celebrarono le ultime Cortes dello Stato assoluto borbonico, mentre l'influenza rivoluzionaria francese si farà sentire nel concreto, insieme alle armi napoleoniche, circa vent'anni dopo, precisamente nel 1808, al momento dello scoppio della Guerra di Indipendenza spagnola contro l'occupante francese.

A tal fine appare necessario esordire con qualche breve considerazione circa l'articolata storia politica delle Cortes, che, come si vorrà sottolineare meglio più avanti, porterà ad alcuni profondi cambiamenti politici, che si avranno con l'operato delle Cortes di Cadice degli anni 1810-1812, sfociato nella costituzione del 1812, di cui, nel 2012, è caduto il bicentenario.

Tuttavia, se appare innegabile l'affermazione che la costituzione, intesa in senso moderno e rivoluzionario sulla scia dell'esperienza politica francese (rectius delle varie esperienze o dei diversi tentativi politici costituenti) della fine del secolo XVIII, rappresenti il vero punto di inizio per una nuova idea di Stato, la foga costituente non cancellerà la storia politico-istituzionale spagnola; anzi le stesse Cortes di Cadice, le prime dopo quelle del 1789 (queste, come detto, le ultime di Antico Regime), sono anche esse concepite sotto diversi aspetti, almeno nei decreti di convocatoria, come assemblea di Antico Regime, pur se con competenze assai gravose e significative.

Conviene ripercorrere, pur sommariamente, la stessa idea medievale e moderna dell'istituzione-Cortes, per meglio comprendere l'importanza delle medesime nel fatidico anno $1789^{1}$.

\section{Paragrafo Primo: Brevi note sulle Cortes di antico regime}

In primo luogo va detto che, pur nelle variegate esperienze dei diritti forali spagnoli $^{2}$ (i fueros ovvero i singoli complessi di norme, essenzialmente non scritte, che

\footnotetext{
${ }^{1}$ Sul punto, con riguardo alle Cortes di Castiglia, si rimanda a testi fondamentali quali F. Martínez Marina, Teoría de las Cortes ó grandes juntas nacionales de los reinos de León y Castilla, Madrid, 1813; V. Piskorski (traduzione a cura di C. Sánchez-Albornoz), Las Cortes de Castilla en el periodo de tránsito de la Edad Media a la Moderna 1188-1520, Barcellona, 1930; J. Valdeón Baruque, Las Cortes de Castilla y León, Valladolid, 1987; ex multis, fra i contributi più recenti si veda l'interessantissimo AA.VV., Las Cortes de Castilla y León, 1188-1988: Actas de la tercera etapa del Congreso Científico sobre la historia de las Cortes de Castilla y León, León del 26 a 30 de septiembre de 1988, 2 vol., Valladolid, 1990.

${ }^{2}$ Ad oggi ancora vigenti a livello locale in ragione dell'art. 149 n. 8 della costituzione spagnola del 1978 (per la fonte si rimanda al Portal del Boletín Oficial del Estado in www.boe.es).
} 
regolavano il diritto — nel suo insieme - nei singoli regni e territori cristiani della Reconquista), l'assemblea delle Cortes (dal latino curtis) rappresentava la riunione dei vari ceti intorno alla Corona. Tale istituzione è presente già prima della nascita della cattolicissima Spagna e, più precisamente, nei concilii visigoti. ${ }^{3}$

In essi, ove le varie funzioni di potere apparivano assai intrecciate, si stabilisce, dopo l'abbandono dell'eresia ariana in favore dell'ortodossia cattolica, una comunanza di governo nell'amministrazione degli affari laici e di quelli ecclesiastici. Infatti ...oficiales de palacio, grandes y señores de la corte, obispos y prelados eclesiásticos, presididos del Príncipe, se juntaban frecuentemente en algunas asambleas ${ }^{4}$.

In tali asambleas si intravede certo l'equilibrio della costituzione mista, anche se bisognerà attendere il Basso Medioevo e l'affermarsi della Reconquista della penisola iberica da parte dei Regni cristiani perché questo paradigma di governo possa affermarsi e prosperare; infatti in alcuni casi si poteva già configurare un esercizio condiviso del potere di gestione della cosa pubblica da parte dei vari ceti, stretti intorno alla figura del Principe.

Guardando sommariamente ai Regni più importanti per la storia politica spagnola ${ }^{5}$, si ricordi come già nel 1188, anno in cui iniziò il suo regno, Alfonso IX abbia celebrato le Cortes in León -dominus Aldefonsus Rex Legionis et Galletie constituit in curia apud Legionem cum archiepiscopo compostelano, et cum omnibus episcopis, magnatibus et cum electis civibus regni sui ${ }^{6}$ -

Occorre dunque accingersi a comprendere come anche per la Spagna (rectius per le Spagne) sia esistita, a partire da un momento molto lontano nel tempo, una limitazione del potere regio, come quella inaugurata, in Inghilterra, con la "imposizione", nei confronti di Giovanni Senza Terra, della Magna Charta del 1215. E questo elemento assolutamente fondante per tutta la storia costituzionale europea non può prescindere, per la Spagna, da alcune date di grandissima importanza, come il 1188, cui si è già accennato ${ }^{7}$.

\footnotetext{
${ }^{3}$ Esattamente nel III concilio, celebrato nel 589 a Toledo, l'abbandono della fede ariana portò alla conversione dei visigoti al cattolicesimo e alla conseguente nascita del connubio tra potere laico e potere religioso.

${ }^{4}$ G.M. de Jovellanos, Sobre la necesidad de unir al estudio de la legislación el de nuestra historia y antigüedades (discorso tenuto in occasione dell'ingresso dell'A. nella Real Academia de la Historia -anno 1780-), in Obras publicadas e inéditas de don Gaspar Melchor de Jovellanos, colección hecha e ilustrada por Cándido Nocedal, vol. 1, 1858, 290, in Biblioteca de autores españoles (BAE), tomo 46. L'A. verrà richiamato per sostenere l'idea storica (e anglofila) della costituizione spagnola, che prevarrà nel maturo Ottocento spagnolo.

${ }^{5}$ Conviene, nel prosieguo, in tale sede richiamarsi, anche per l'importanza storica rivestita, alle vicende della Corona di Castiglia e a quelle della Corona di Aragona.

${ }^{6}$ Manuel Colmeiro (a cura di), Cortes de los Antiguos Reinos de León y Castilla (por Real la Academia de la Historia), Madrid, vol. I, 1861, 39.

${ }^{7}$ Sul punto E. Mitre Fernández, «A ochocientos años de las ¿primeras? Cortes hispánicas (León 1188) mitos políticos y memoria histórica en la formación del parlamentarismo europeo», Mayurqa: revista del Departament de Ciències Històriques i Teoria de les Arts, nº 22, 1, 1989, 415-426.
} 
Questa storia costituzionale inizia proprio nel 1188 e forse appare poco indagata, giacché il contesto europeo è rivolto ad altri modelli di più larga fortuna; da subito bisogna però distinguere tra le Cortes medievali, quelle borboniche del secolo dell'Assolutismo, il XVIII, e quelle dell'epoca costituzionale ottocentesca.

Proprio l'anno 1188 rappresenta per le Cortes una data di grande rilievo con l'inizio della presenza stabile nella curia regia di alti esponenti laici ed ecclesiastici. Senza dimenticare tuttavia l'esistenza di Cortes in epoca precedente, a Nájera nel 1137, a Toledo nel 1157 e a Burgos nel $1169^{8}$.

Nel 1188 Alfonso II, re di Aragona e conte di Barcellona, riuniva i nobili nella città di Huesca; ma già quasi un secolo prima, nel 1068, Don Ramón Berenguer I aveva convocato bisbes, prelats, ma anche barons, nobles, cavallers, e infine homens de vilas, come anche i ciutadans, secondo quanto si legge negli Usatges de Barcelona, primo código consuetudinario catalano, promulgato sotto lo stesso Don Ramón Berenguer, testo con chiare radici nel diritto visigoto e nel diritto romano (tale sistema normativo avrà successivamente una profonda influenza in Aragona, anche se le Cortes aragonesi — va ricordato- annoveravano due bracci nobiliari).

Il peso lato sensu politico ${ }^{9}$ dell' Estado Llano o General (il Terzo Stato), che non godeva dei privilegi degli altri due estamentos, quello nobiliare e quello ecclesiastico, sarà destinato ad essere sempre più determinante se guardiamo alla Castiglia; anzi la presenza dello stesso potrà dirsi costante a partire da una data ben precisa (con ripercussioni fino alle ultime Cortes di Antico Regime per la Castiglia e la Aragona ovvero quelle celebrate a Madrid nel 1789), se si escludono le convocazioni in ragione di finalità meramente cerimoniali.

In tal senso si pensi ad una delle epoche di massimo splendore per la Spagna ovvero la prima metà del secolo XVI. I tre stati vennero convocati a Toledo dall'Imperatore Carlo V e svolsero i loro compiti tra la fine del 1538 e l'inizio dell'anno successivo. I tre bracci si riunirono separatamente ed emerse, assai decisa, l'opposizione della nobiltà all'introduzione della sisa, una nuova tassa (dal fr. ant. assise). Da una parte il clero non abbracciò una decisone netta, data la natura "temporale" dell'imposta; tuttavia, anche grazie all'influenza dell'Arcivescovo di Toledo, il Cardinale Juan Tavera ${ }^{10}$, che presiedeva le Cortes, il clero non prese le parti della nobiltà.

La nobiltà rimase ferma sulla sua posizione e nel gennaio del 1539 fu letteralmente «liquidata», risultando estromessa, per volontà sovrana, dalle Cortes di Toledo ${ }^{11}$.

Anzi non sarà più convocata, in modo che nelle stesse siederanno solo i procuradores de las ciudades y villas de voto en Cortes, che, in molte occasioni, non potranno

\footnotetext{
${ }^{8} \mathrm{Ivi}, 416$.

${ }^{9}$ Termini come "politico" sono in questo studio usati con una certa "cautela" e lontano da ogni significato modernamente inteso in senso pieno.

${ }^{10}$ Già esponente di spicco del Consejo de Castilla, vero centro del potere della Monarchia spagnola nell'epoca moderna.

${ }^{11}$ Cfr., F. Martínez Marina, Teoría de las Cortes, 77.
} 
essere veramente liberi nell'esercizio delle proprie funzioni, in quanto soggetti ad una sorta di mandato imperativo da parte delle comunità di provenienza, ma anche, e soprattutto, all'influenza regia.

$\mathrm{Ma}$, andando con ordine, occorre ricostruire, pur brevemente, la vicenda bassomedievale delle Cortes, con particolare riguardo a quelle della Castiglia ${ }^{12}$.

Come ricordato, nel 1188 Alfonso IX convocò per il regno di León i rappresentanti dell'Estado Llano perché potessero far sentire la loro voce al pari degli estamentos privilegiati.

Appare lecito pensare a questo momento come a un caposaldo del costituzionalismo spagnolo, seppur nel solco dell'idea ancora medievale ovvero pactada (tra i diversi ceti) della res publica e della stessa concezione del potere.

La questione ruota peraltro sempre intorno ad un punto fondamentale ovvero se la voce dei vari procuradores (in particolar modo di quelli dell'Estado Llano) trascenda l'appartenenza cetuale degli stessi. Ma a tale problematica si vuole ora solo accennare. Va tuttavia ricordato - riprendendo le linee del discorso principalecome tali procuradores fossero soggetti alle direttive delle comunità da cui provenivano, quasi gli stessi fossero meri nuncii delle stesse.

In tal senso va ricordato il dovere per tali procuradores di portare all'attenzione del principe i c.d. cuadernos de peticiones.

Venendo alla composizione delle Cortes, in primo luogo il diritto di sedere nelle stesse spettava generalmente agli esponenti degli estamentos privilegiati, come nella primordiale esperienza visigota; tuttavia tale composizione per la Castiglia, che in epoca moderna comprendeva ormai da tempo anche il regno di León e, nel secolo XVIII, i rappresentanti dell'Aragona, varierà in modo assai significativo.

Dapprima le Cortes nascono infatti intorno alla Corona, che le convoca, e trattano affari spesso riguardanti la stessa, ma anche, in definitiva, la comunità tutta; basti pensare al nodo della tassazione e dei tributi, mai sufficienti nel periodo della Reconquista contro l'occupante arabo.

E le finanze, secondo una regola non scritta e di larga applicazione anche fuori dalla penisola spagnola, gravavano sul Terzo Stato.

Così in Castiglia non si arrivò a convocare il braccio ecclesiastico tra il 1209 e il 1301 e in seguito tale sorte spettò al braccio nobiliare tra il 1480 e il 1505, a differenza dei rappresentanti dell'Estado Llano (il numero di due membri per ogni comunità rappresentata era di regola di due procuradores e rimarrà fermo fino al 1789, anno delle ultime Cortes di antico regime in Castiglia), i soli convocati.

\footnotetext{
${ }^{12}$ Va subito ricordato come le competenze delle Cortes trovarono un loro primo fondamento nella consuetudine, che a fatica andò decadendo sotto l'opera legislativa accentratrice della Corona di Castiglia. A riguardo si pensi a come già il diritto castigliano del secolo XIII configurasse in capo al principe il pieno potere di dettare norme (Partida Primera, titolo primo, legge XII). Per il testo delle Partidas si rimanda all'edizione dell'1807 a cura della Real Academia de la Historia (in Biblioteca Virtual Miguel de Cervantes in http://www.cervantesvirtual.com).
} 
E, pur in presenza di un obbligo giurato per il principe di convocare le Cortes entro un certo intervallo di tempo prestabilito, le stesse sono difficilmente inquadrabili nel concetto di Parlamento moderno, anche se il percorso del costituzionalismo spagnolo di Antico Regime presenta alcune peculiarità rispetto ai modelli europei ben più noti (si ricordi il lungo sonno, quasi bicentenario, degli Stati Generali francesi, che si riuniranno per l'ultima volta nel 1789).

E se va sottolineata la inviolabilidad dei procuradores, va aggiunto che gravava sulle comunità di provenienza il c.d. salario de procuración; va poi menzionato, come detto, una sorta di "mandato imperativo" delle stesse, nonchè l'oggetto, spesso limitato, della regia convocatoria a Cortes.

Le competenze, diverse e variegate, anche in ragione delle diverse convocatorias o di norme non scritte, apparivano dunque incerte, in modo che le cartas rappresentassero un vero e proprio memorandum per il principe, una volta terminate le riunioni delle Cortes.

Tali competenze, che in definitiva coprivano le varie funzioni della Curia regis (non ultime quelle in tema di giustizia), risultavano allora indubbiamente intersecarsi nell'articolato sistema di rappresentanza delle Cortes.

Tuttavia, si ricordi, l'accentramento regio, già operante alla metà del secolo XIII grazie all'opera legislativa del re Alfonso X il Saggio (in particolare con la redazione delle Siete Partidas), continuerà fino al consolidarsi della Monarchia cinquecentesca (creata dai Re Cattolici, Ferdinando di Aragona e Isabella di Castiglia) e di quella dei Borbone (secolo XVIII), sempre lungo la linea volta a dare uno stabile quadro istituzionale sotto il crescente peso della Corona; in tal senso la consuetudine e in particolare i diritti forali, alla base delle prerogative delle Cortes dei singoli Regni (e in particolare ai rappresentanti nelle stesse del Terzo Stato), dovranno cedere il passo rispetto alle disposizioni emanate dalla Corona, in modo tale che i vincoli per i procuradores finiranno per discendere dalla fedeltà verso la stessa più che dal legame con le comunità locali.

Et porque al Rey pertenesce, è hà poder de facer fueros, è Leys, è de las interpretar, è declarar, è emendar dò viere que cumple, tenemos por bien que si en los dichos fueros, ó en los libros de las Partidas sobredichas, ò en este nuestro libro, ò en alguna, ò en algunas Leys de las que en el se contienen, fuere menester interpretacion, ò declaracion, ò emendar, ò annadir, ò tirar, ò mudar, que Nos que lo fagamos.

Siamo a metà nel secolo XIV e così si può leggere nella prima legge del titolo ventottesimo dell'Ordenamiento de Alcalá, insieme di leggi raccolte da Alfonso XI nel $1348^{13}$.

${ }^{13}$ Nell'edizione a cura di I.J. de Asso y del Río e M. de Manuel y Rodríguez (Madrid, 1776, 72-73). 
Va peraltro ricordato, in senso opposto, quanto stabilito dalle leggi prima e seconda del titolo settimo del libro sesto della Nueva Recopilacion del 1567, che menzionavano come necessario il consenso dei rappresentanti delle ciudades e delle villas al fine della imposizione di tributi.

\section{Paragrafo Due: le ultime Cortes di Antico Regime}

All'inizio del secolo XVIII, con il consolidarsi del potere di Filippo V (con precisione nel momento della conclusione della Guerra di Successione spagnola negli anni 1713-14), l'incipiente assolutismo regio è volto a svuotare le Cortes, riunione dei procuradores delle ciudades e delle villas (ovvero comunità che godevano di determinati privilegi) con diritto di voto nelle stesse, di ogni potere concreto, nel contesto del tramonto della concezione basso-medievale della sovranità compartida tra Corona e Cortes, che pur era sopravvissuta all'epoca del passaggio dalla Reconquista a quella dei Re Cattolici, Isabella e Ferdinando, all'inizio del secolo XVI.

Tuttavia Carlo IV, alla morte del padre, Carlo III, nel 1788, si sentì in dovere di convocare le Cortes ${ }^{14}$ perché prestassero giuramento di fronte al figlio Ferdinando, nuovo erede al trono; si trattò pur sempre di un aspetto cerimoniale, anche se non totalmente vuoto di significato.

Va ricordato che le riunioni delle Cortes, nel secolo XVIII, rappresentarono l'occasione per sontuosi e fastosi cerimoniali ${ }^{15}$, mentre le súplicas o peticiones alla Corona si presentavano come lo strumento attraverso cui le ciudades e le villas esprimevano il desiderio che la Corona intervenisse in questioni che riguardavano le stesse direttamente.

Tuttavia una certa clausola aperta era presente nella convocatoria del 22 maggio $1789^{16}$, inviata all'attenzione delle ciudades e delle villas con un atto della Camara de Estado in data 31 maggio dello stesso anno ${ }^{17}$.

\footnotetext{
${ }^{14}$ In tal senso Carlo IV riconfermava un'antica tradizione che vincolava i re di Castiglia nel caso di successione al trono. Tale norma non scritta, in questo caso, prevaleva rispetto alla logica dell'assolutismo e della sua forma accentratrice, che si manifestava anche attraverso lo strumento di normazione unilaterale nelle mani della Corona. Circa l'obbligo, per la Corona, di convocare le Cortes in Castiglia nel momento della successione al trono si rimanda a F. Martínez Marina, Teoria de las Cortes, Madrid, 1813, 32. Tuttavia Ferdinando VI, figlio di Filippo V, non convocò mai le Cortes durante gli anni del suo regno (1746-59). Va subito detto, circa le Cortes del 1789, che le stesse comprendevano anche i rappresentati delle ciudades e villas aragonesi con diritto di voto, giacché le stesse Cortes aragonesi erano scomparse a seguito della politica accentratrice assolutista di Filippo V, attuata nel corso delle prime due decadi del secolo XVIII e culminata nella emanazione dei Decretos de Nueva Planta (tuttavia le Cortes continueranno a riunirsi in Navarra fino agli trenta del secolo XIX).

${ }^{15} \mathrm{Cfr}$. G. Desdevises du Dézert, L'Espagne de l'ancien regime. Institutions, Parigi, 1899.

${ }^{16}$ Per gli atti delle Cortes del 1789 si rimanda a la Colección de documentos inéditos para la historia de España, tomo XVII, a cura di M. Salvá e P. Sainz de Baranda, Madrid, 1850, 5-455.

${ }^{17}$ J. Longares Alonso, Las últimas Cortes del Antiguo Régimen en España (19 septiembre-5 diciembre de 1789), Revista de historia moderna, n. 3, 1974, 113.
} 
Infatti, oltre che per il necessario cerimoniale nel contesto della successione, si convocava la riunione delle Cortes per trattare altri e diversi negocios si se propusieron y pareciese conveniente resolver ${ }^{18}$.

Tuttavia, in concomitanza degli eventi rivoluzionari francesi $i^{19}$, queste parole, ora riportate, avrebbero potuto rappresentare la via legale per istanze di riforma, non certo rivoluzionarie, ma comunque assai profonde ${ }^{20}$.

Dopo che i primi procuradores erano arrivati a Madrid già ad agosto, il 14 di settembre, sotto la presidenza di Campomanes, Gobernador del Real Consejo de Castilla, e futuro Presidente delle Cortes del 1789, iniziano i preparativi, tra cui la presentazione delle credenziali dei procuradores dell'Estado Llano; cinque giorni dopo, il 19, si aprono le Cortes alla presenza di Carlo IV e il 23 si tiene il giuramento di fedeltà dei tre Stati nel Real Monasterio de San Gerónimo.

Sette giorni dopo si apre la prima sessione delle Cortes $^{21}$ e i lavori entrano nel vivo.

Si ricordi che la fedeltà dei procuradores delle ciudades va alla Corona e che gli stessi non sono vincolati da alcuno né da eventuali "mandati imperativi" ricevuti in sede di elezione o nomina ${ }^{22}$ (la quale poteva avvenire anche attraverso sorteggio).

L'operato delle Cortes del 1789 rimane di certo immune dal fervore rivoluzionario, che infiamma la vicina Francia nell'estate dello stesso anno. Non solo per volontà dei procuradores dell'Estado Llano o General (l'unico -si ricordi- rappresentato), ma anche in ragione del fatto che gli stessi membri delle Cortes sono pur sempre attori di quella legalidad fundamental che le Cortes, al pari della Corona, sono chiamate a difendere; ben diversa la situazione in Francia, dove il Terzo Stato si autoproclama prima Assemblea nazionale e poi Assemblea costituente, quasi a volersi rifare di un sonno imposto ex auctoritate per quasi due secoli.

Le Cortes si muovono invece ancora in un complesso sistema amministrativogiurisdizionale, più che politico, nel quale i poteri e le funzioni si intrecciano nella rete del c.d. régimen polisinodial. Tale si presentava l'articolazione centrale (in primo

\footnotetext{
${ }^{18}$ Certo non era la prima volta che si inseriva tale clausola nella convocatoria (cfr. ivi, 115); per la citazione v. Colección de documentos, 8.

${ }^{19}$ Sempre nel maggio del 1789, il 5 per la precisione, si erano inaugurati i lavori degli Stati Generali alla presenza di Luigi XVI e del suo ministro delle finanze Jacques Necker.

${ }^{20} \mathrm{E}$ i dibattiti certo non erano sopiti; si pensi alla discussione circa la riforma della legislazione agraria (sul punto Jovellanos, uno dei più grandi esponenti dell'Illuminismo spagnolo, scriverà un celebre Informe nel 1795) e alla espulsione dei Gesuiti nel 1767.

${ }^{21}$ J. Longares Alonso, Las últimas Cortes del Antiguo Régimen en España, 114-115.

${ }^{22}$ Trentasette erano le comunità aragonesi e castigliane rappresentate. Non sfugga come tra i rappresentanti dell'Estado llano (l'unico chiamato nelle Cortes, eccezion fatta per le fasi cerimoniali) figurino procuradores di diversa estrazione sociale, tra cui molti soggetti dotati di un titolo nobiliare. Ci si chiede se si tratti di nobili decaduti o di emulatori di Publio Clodio Pulcro, che, grazie alla transitio ad plebem, potette accedere nel 58 a.c. alla carica di tribuno della plebe ( $\mathrm{M}^{\mathrm{a}}$. R. Prieto, Las Cortes de 1789, (estratto della tesi dottorale), Madrid, 1973, 10 ss).
} 
luogo il Real Consejo de Castilla) al pari di quella locale (Juntas variamente denominate) della Monarchia assoluta borbonica nel secolo XVIII, apparato, comunque, in parte ereditato dalle esperienze ordinamentali dei preesistenti regni.

Tuttavia tale "costituzione politica" settecentesca non era certo immune da cambiamenti. Circa le Cortes del 1789 va infatti evidenziato un dato storico:

Le Cortes vengono convocate da Carlo IV per ripristinare l'ordine successorio stabilito dalle Partidas $(2,15,2)$ e abrogato da Filippo $V^{23}$ : sotto la Monarchia assoluta, infatti, tale ley fundamental poteva essere modificata solo con il consenso dei rappresentanti estamentales.

Pur in presenza di uno Stato assoluto, se non una norma scritta, almeno l'opportunità politica e una diffusa opinione pubblica legittimano dunque la convocazione delle Cortes per modificare la legge di successione, già emendata sotto il regno di Filippo V (1700-1746).

Tuttavia, come accennato, nel periodo che intercorre tra la convocazione delle Cortes e la riunione delle stesse (maggio-metà settembre del 1789), la Rivoluzione ha tempo di scoppiare e di avere una prima propagazione in Francia.

Viene infatti decretata, agli inizi di agosto, l'abolizione dei diritti feudali e, alla fine dello stesso mese, vengono solennemente stabiliti diritti universali nella celeberrima Dichiarazione dei diritti dell'uomo e del cittadino.

Le Cortes che a settembre si riuniscono a Madrid non possono non risentire profondamente di tali avvenimenti, atteso l'impatto degli stessi in una società, che, al pari della corte regia, risulta profondamente afrancesada nella cultura e nei costumi; ma lo stesso Illuminismo spagnolo non avrebbe potuto mai giustificare un simile stravolgimento istituzionale.

Il governo spagnolo non tardò invero a percepire l'importanza degli accadimenti francesi; e il Conte di Floridablanca, al tempo Secretario del Despacho de Estado (i. e. per gli Affari esteri) mostra da subito, nelle parole rivolte al suo ambasciatore a Parigi, il conte di Fernán Nuñez, un netto atteggiamento di condanna verso gli eventi francesi ${ }^{24}$.

Per Floridablanca l'Illuminismo ha contribuito dunque alla conquista di diritti in nome della felicità, ma, allo stesso tempo, ha cancellato quel senso di stabilità che caratterizzava l'antico regime.

\footnotetext{
${ }^{23}$ M. Friera Álvarez e I. Fernández Sarasola, Contexto histórico de la Constitución española de 1812, par. 2 (El Gobierno de Carlos IV y Godoy) in http://bib.cervantesvirtual.com/portal/1812/.

${ }^{24}$ Yo condeno estas cosas y compadezco al buen Rey, a sus ministros y a ese pueblo ligero, incostante y aventurero. Se dice que el siglo ilustrdo ha instruido al hombre de sus derechos. Pero le ha quitado con la verdadera felicidad el reposo y la seguridad de su persona y de su familia. Nosotros no querémos aqui tanta ilustración, ni lo que trae como consecuencia: insolencia de los actos, de las palabras y de los escritos contra los poderes legítimos...”, R. Menéndez Pidal, (fundador), Historia de España, tomo XXXI, volumen II, La época de la Ilustración: Las Indias y la Política exterior, 1988, Madrid, 452 (le parole "siglo ilustrado" sono già riportate in corsivo).
} 
Da parte sua, Carlo IV ebbe modo di conoscere gli eventi del 1789 anche grazie alla lettera che Luigi XVI gli indirizzò il 7 di ottobre: il re francese scriveva di sentirsi privato della sua libertà, in modo che gli atti e le disposizioni da lui emanati dal giorno seguente alla presa della Bastiglia erano da considerarsi nulli.

I rapporti con la Francia, così da sempre vicina in tutti i sensi, risultavano regolati dai pactos entre Reyes, conclusi lungo il Settecento e volti a confermare l'alleanza tra Francia e Spagna, entrambe governate dai Borbone.

Ma di fatto rimane pur sempre la considerazione che nelle relazioni tra le due nazioni, divise dai Pirenei, godeva di una certa posizione preminente la Francia, come già si era manifestato durante il secolo XVIII in campo culturale e politico.

E tale influenza permaneva, per cui, già alla metà dell'ottobre del 1789, vennero diffusi preoccupati despachos affinché si procedesse al controllo delle frontiere, al fine di evitare il diffondersi della propaganda rivoluzionaria.

Inoltre una determinazione reale del 21 di Settembre sollecitò al Sant'Uffizio la raccolta tutti i documenti directa o indirectamente opuestos a la subordinación, vassalaje, obediencia y reverencia debidos a nuestro venerado monarca y al vicario de Jesucristo, por ser tales ideas antievangélicas y expresamente opuestas a las doctrinas de los santos apóstoles Pedro y Pablo ${ }^{25}$.

Negli stessi giorni le Cortes riunite a Madrid vennero chiuse in modo precipitoso (23 ottobre) a un mese dalla loro apertura.

Nelle stesse non era mancata la volontà di presentare petizioni al re, anche in considerazione di un ampliamento, una extralimitación dei poteri dei procuradores, grazie alla rappresentazione di altri eventuali negozi da trattare.

Nelle Cortes si poteva, allo stesso tempo, riscontrare un'opposizione passiva a riforme troppo decise; Miguel Artola ricorda:

En la sesión del 13 de octubre se pidió al conde de Campomanes, gobernador del Consejo y presidente de las Cortes, hiciese presente a S. M. el deseo del Reino de dirigirle peticiones... En la siguiente sesión, celebrada cuatro días mas tarde, les fue respondido que, como el tiempo está adelantado y de la duración de las Cortes resultan gastos a las ciudades y perjuicios a los caballeros procuradores, sus representantes, se hace preciso finalizar cuanto antes este Congreso, y en lugar de presentar súplicas o peticiones, Su Majestad permite que, disueltas éstas, puedan cada una de las ciudades y villas de voto en Cortes, representar separadamente cuanto estimen conducente al mejor servicio de $S$. M. y servicio público ${ }^{26}$.

Le Cortes vengono dunque chiuse in modo precipitoso il 23 Ottobre del 1789; le loro sessioni erano state dominate da temi di grande rilievo, in un generale clima di

\footnotetext{
${ }^{25}$ R. Menéndez Pidal, op.cit., 457.

${ }^{26} \mathrm{Ivi}, 458$.
} 
diffidenza verso gli eventi rivoluzionari francesi: quello della modifica della legge di successione al trono e il dibattito circa la lotta alla manomorta.

Ma cerchiamo di comprendere un aspetto più propriamente dottrinario.

In una prima approssimazione va allora riproposta la fondamentale differenza tra Illuminismo francese e Ilustración: il primo si manifesta in modo drastico e rivoluzionario, con la conseguente rottura dei legami con l'Antico Regime; il secondo si muove lungo la linea della conservazione delle fondamenta politiche della società, pur in presenza di nuove aspirazioni per la comune utilità e per la pubblica felicità.

L'Ilustración, circa la forma di governo da instaurare, presenta un'indole decisamente controrivoluzionaria; anzi appare più corretta la tesi che la stessa perseguisse una forma di governo da "conservare" piuttosto che da "instaurare", seppur con i dovuti accorgimenti.

Tale affermazione trova riscontro nell'autorevole opinione di Montesquieu, secondo il quale "gli Spagnoli e i Portoghesi sono ancora sotto tutela in Europa"; e la Monarchia borbonica in particolare appariva di certo legata ad un ambiente cattolico ortodosso, in cui l'Inquisizione, al servizio — si ricordi- della stessa Monarchia, esercitava un profondo controllo culturale.

Sul punto, secondo Padre Feijoo, filosofo benedettino, uno dei più importanti pensatori dell'Illuminismo spagnolo, il credo cattolico doveva certamente essere sottratto all'ambito dell'opinabile; ma allo stesso tempo, come egli propugnava, era possibile una separazione tra scienza e teologia, e, in definitiva, tra fede e ragione; e su un piano politico, nella metà del Settecento, appariva ormai chiaro che il programma politico ilustrado comprendeva vari campi di azione (economia, istruzione, desamortización.... $)^{27}$.

Ma era altrettanto chiaro che tale ambizioso programma di cambiamenti sociali ed economici e di rinnovamento dell'amministrazione regia non avrebbe mai portato a porre in discussione le basi dell'ordinamento, ovvero la società estamental, il confessionalismo cattolico e l'assolutismo monarchico (anche se quest'ultimo appariva come un novum politico settecentesco, in rottura con l'antica costituzione medievale consuetudinaria e pattista).

In una parola, nonostante il consolidarsi dello Stato assoluto, la dialettica CoronaCortes rimaneva aperta, nonostante gli equilibri si sbilanciassero in favore della prima, la quale andava accentrando in sè tutta la sovranità, che, prima del Settecento e fin da epoca risalente (inizio del Basso Medioevo), era divisa (compartida) tra la Monarchia e gli ordini estamentales. Questo equilibrio, infatti, se venne realmente turbato dai fatti francesi del 1789, era già stato incrinato dall'instaurarsi della Monarchia assoluta borbonica nel secolo XVIII.

\footnotetext{
${ }^{27}$ Né mancavano scambi culturali; le opere di Campomanes, Presidente delle Cortes del 1789, trovano diffusione nella divisa penisola italiana, mentre Muratori, Filangieri e Beccaria godono di fortuna in Spagna.
} 
I cambiamenti politici erano dunque già presenti nella storia spagnola; la stessa linea accentratrice inaugurata con i Decretos de Nueva Planta a inizio del secolo XVIII aveva infatti intessuto l'ordinamento in un modo assai diverso da quanto era avvenuto con la politica di Luigi XIV in Francia; colà, infatti, la presenza, a differenza del caso spagnolo, di un soggetto politico unitario, aveva facilitato la nascita dello Stato assoluto, che, diversamente, in Spagna dovette tener in conto delle varie "Spagne", eredi dei singoli regni delle Reconquista.

Se dunque la Rivoluzione Francese condizionò, in qualche modo, l'operato delle Cortes spagnole, lo stesso può dirsi anche con riferimento ad altre realtà, come quella inglese, circa alcune importanti conquiste politiche che si avranno solo con l'epoca vittoriana (iniziata nel 1837). E a tale riguardo si ricorda il definitivo affermarsi della forma di governo parlamentare ${ }^{28}$. Infatti proprio negli anni tra il 1789 e l'ascesa di Napoleone il ricordo, ancora troppo vicino, degli eventi rivoluzionari, nonchè la minaccia delle armate francesi, porterà molti pensatori politici a rivedere le proprie posizioni riformatrici, come nel caso di Edmund Burke ${ }^{29}$, che si può definire vero campione del pensiero controrivoluzionario, ma non certo un fermo reazionario come, ad esempio, Joseph de Maistre nell'epoca della Restaurazione.

Ma torniamo alla fattispecie spagnola: le parole che seguono sembrano riassumere il quadro politico delle Cortes del 1789, spesso offuscate dall'operato del Terzo Stato in Francia nello stesso anno.

Si può infatti leggere:

De todo lo referido, se deduce que las Cortes en Castilla no pueden juntarse si el Reino las convoca, que su celebración es arbitraria en los soberanos de España, que ni ahora ni en tiempo alguno ha tenido el Reino junto en Cortes autoridad alguna legislativa. Que sólo puede el Reino proponer y suplicar. Que los tres brazos eclesiástico, noble y popular quedaron reducidos desde el año de 1538 a las ciudades y villas de voto en Cortes. Que a las Cortes en que se tratan asuntos particulares sólo concurren los procuradores de las ciudades y que aún a los actos de juras es arbitrio de Su Majestad la clase y número de personas que han de concurrir. ${ }^{30}$

Si tratta certo di un quadro oramai dominato dall'assolutismo borbonico, che, tuttavia, rappresenta solo l'ultima fase di una storia costituzionale, quella spagnola, se-

\footnotetext{
${ }^{28}$ Basti pensare, che un primo significativo allargamento della base elettorale si avrà in Inghilterra con la riforma del 1832.

${ }^{29}$ Autore dell'opera Riflessioni sulla Rivoluzione francese (1790), in cui la difesa della tradizione è da strada per la condanna della vis rivoluzionaria francese che professa, per Burke, ideali essenzialmente astratti.

${ }^{30}$ Noticia sobre el origen y presidencia de las Cortes de León y Castilla desde 1202 (Archivo Histórico Nacional (AHN), Estado, leg. 3.382-2, $\mathrm{n}^{\mathrm{o}}$ 15); riportato in F. Lorenzana de la Puente, La representación política en el Antiguo Régimen. Las Cortes de Castilla, 1655-1834, (tesi dottorale), volumen II, Cáceres, 2010, 203.
} 
gnata da un evento accaduto due secoli e mezzo prima, ovvero l'eliminazione della presenza degli estamentos privilegiati dalle Cortes di Castiglia che da quel momento continuarono tuttavia a trattare alcuni gravi temi, quali la tassazione (in particolare straordinaria).

Rimaneva certo integra l'influenza della Corona nelle Cortes, e anche più in generale l'alleanza con la parte più importante dell'Estado Llano. Questo ultimo manteneva la sua diffidenza verso gli stati privilegiati e la Corona, con i quali, dalla metà del secolo XVI, non occuperà più il campo del confronto, ma solo i banchi del cerimoniale, come nel caso del giuramento nel momento della successione al trono.

Questione, quella della successione al trono, che rimaneva ancora aperta anche dopo la fine dell'assolutismo nel 1833, con la morte di Fernando VII.

E la mancata pubblicazione, per volontà di Carlo IV, della pragmatica ${ }^{31}$, approvata dalle Cortes del 1789, che abrogava la legge salica di successione al trono (introdotta con l'Auto Acordado 32 del 1713 sotto il regno di Filippo V), avrebbe portato, quarant'anni dopo, a gravi conseguenze.

Infatti la tardiva pubblicazione della Pragmatica da parte di Ferdinando VII, nel 1830, favorirà sua figlia, la futura Isabella II, nata in quello stesso anno, che la storia conoscerà come la "Regina bambina", a discapito del fratello di Ferdinando VII, don Carlo, aspirante al trono di Spagna con il nome di Carlo V.

Si potrebbe configurare come una mera questione procedurale la mancata tempestiva pubblicazione della pragmatica del 1789; tuttavia, a ben vedere, le conseguenze, anche in considerazione di altri fattori, in concomitanza con il definitivo tramonto dell'Antico Regime, saranno gravi ${ }^{33}$.

La guerra che ne nascerà e che vedrà contrapposte le fazioni liberali di Isabella II ed i partidarios di chiara indole conservatrice del sedicente Carlo V contribuirà ad aprire la strada a diversi tentativi costituzionali nel corso degli anni subito successivi al 1833.

E in primis ciò avvenne con l'Estatuto Real ${ }^{34}$ concesso nel 1834 da Maria Cristina, moglie del defunto Ferdinando VII, in qualità di reggente della loro figlia Isabella II; il testo appare legato alla tradizione, ma anche al rinnovamento, con l'introduzione di un sistema bicamerale.

Delle due assemblee una era destinata agli stati privilegiati (i cui membri erano chiamati próceres $^{35}$ ) ed una a quello popular (i cui membri erano chiamati procura-

\footnotetext{
${ }^{31}$ Decisione dovuta anche a circostanze contingenti, come lo scoppio della Rivoluzione in Francia ( $\mathrm{M}^{\mathrm{a}}$. Rosario Prieto, Las Cortes de 1789, 10).

${ }^{32}$ In tale caso da intendersi nell'accezione di atto normativo.

${ }^{33}$ Altra questione solo apparentemente procedurale si ebbe già nel 1789 con il tentativo del Terzo Stato francese di abolire la votazione per Stati: ciò contribuirà all'abbandono da parte dello stesso dell'assemblea di Versailles in modo che, da quel momento, potrà parlarsi di un significativo punto di cesura costituzionale e politica.

${ }^{34}$ Per il testo v. http://www.dircost.unito.it.

${ }^{35}$ Artt. 3 ss.
} 
dore $^{36}$ ); da ultimo una grande innovazione era rappresentata dalla menzione nel testo (e dal conseguente riconoscimento costituzionale) - sic! - della figura del Presidente del Consejo de Ministros ${ }^{37}$.

Lo svolgimento dei lavori delle Cortes del 1789 non dovrebbe dunque essere oggetto di interesse per il solo fatto della - casuale- concomitanza con lo scoppiare della Rivoluzione francese.

Né si dovrebbe leggere questo ultimo evento solo in un'ottica volta ad offuscare il quadro istituzionale della Spagna del secolo XVIII.

Se infatti il 1789 rappresenta l'anno zero per la Francia, che esce dall'assolutismo, nello stesso anno lo scoppio del "big bang" rivoluzionario deve ancora travalicare $\mathrm{i}$ confini pirenaici.

Solo successivamente il conflitto intestino nella Francia monarchica si trasformerà infatti in una guerra di espansione per la Prima Repubblica francese, nata nel 1792.

Epoca, quest'ultima, che la Spagna vedrà sempre da lontano; solo negli anni dell'Impero, precisamente nel 1808, la minaccia francese si rivolgerà contro la Spagna, inizialmente alleata alla volta della conquista del Portogallo.

Ne scaturirà una guerra tra l'Impero napoleonico occupante e la Spagna levantada e insorta, che logorerà le armate napoleoniche per ben sei anni (fino al 1814), ancor prima della campagna di Russia (1812).

Nel corso di questa lunga lotta per qualche anno sembrarono risorgere molte delle voci dell'Illuminismo e del dispotismo illuminato spagnoli in cerca di un modello politico per la Spagna levantada. Molti di quei notabili, fedeli alla Monarchia borbonica, come anche le voci più exaltadas dell'Estado Llano, possono ora mettere in atto molte di quelle istanze di riforma fino a quel momento solo ipotizzate. Infatti molti gentiluomini, come il Conte di Floridablanca, figli di quel mondo barocco del secolo XVIII, vengono chiamati in prima linea a confrontarsi con personaggi di formazione più tipicamente borghese, tra i quali va ricordato Lorenzo Calvo de Rozas, commerciante, a Madrid, dalle grandi fortune, che in quegli anni lottò per riconoscimento della libertà di stampa e per l'adozione di decise riforme costituzionali.

Entrambi opereranno, seppur in diversi momenti, nella Junta Suprema Central, che tra il settembre del 1808 e il gennaio del 1810 governò la Spagna, in qualità di autorità reggente per conto di Ferdinando VII, trattenuto con la forza per volontà del Bonaparte nel castello di Valençay per tutto il corso degli anni della Guerra di Indipendenza spagnola contro l'occupante francese ${ }^{38}$.

Proprio tale Junta opera la transizione; ma questa come avviene? Le forme antiche segnano la strada per profondi cambiamenti.

\footnotetext{
${ }^{36}$ Artt. 13 ss.

${ }^{37}$ Come all'art. 26.

${ }^{38}$ Per un quadro generale di quegli anni e circa i cambiamenti politici posti in atto in tale periodo v. S. Scandellari, Da Bayonne a Cadice : il processo di trasformazione costituzionale in Spagna: 1808-1812, Messina, 2009, passim.
} 
Riunite, infatti, le Cortes di Cadice, nel settembre 1810, le stesse vengono chiamate a "modernizzare" le forme politiche, ma non a stravolgerle. In una parola esse devono riscoprire la verdadera constitución, quella storica, che non può dirsi certo dimenticata $^{39}$, ma solo sospesa nella sua vigenza o effettività. Tutto questo in un contesto da migliorare, non da stravolgere, giacché certe influenze "rivoluzionarie" portano con sè anche gravi germi di instabilità.

Come non pensare, ad esempio, alla pretesa dell'Assemblea gaditana, sulla scia dell'operato del "governo" della Convenzione, di fare da guida politica e di rendere la Regencia (subentrata ad inizio del 1810 alla Junta Suprema Central) un mero organo esecutivo della volontà delle Cortes?

$\mathrm{Ma}$, come accennato, queste ultime non dovevano, almeno nelle intenzioni iniziali, riunirsi per dare sfogo al loro spirito exaltado, che approderà al testo della costituzione del 19 marzo 1812, di cui si è celebrato l'anno il bicentenario.

Vi è allora di più. In primo luogo va ricordato come l'assemblea gaditana degli anni 1810-1812 abbia operato con un fine ben preciso. Infatti:

Las Cortes generales y extraordinarias de la Nación española, bien convencidas, después del más detenido examen y madura deliberación, de que las antiguas leyes fundamentales de esta Monarquía, acompañadas de las oportunas providencias y precauciones, que aseguren de un modo estable y permanente su entero cumplimiento (...).

Così può leggersi nel preambolo che precede il lungo articolato della costituzione del $1812^{40}$.

Le leggi fondamentali della Monarchia, preesistenti allo stesso Settecento borbonico, devono essere corrette in modo che in quel preciso momento storico (e in futuro) possano ritrovare la vigenza che hanno perso; le stesse sono dunque preesistenti e devono essere solo acompañadas de las oportunas providencias y precauciones.

In tal senso si comprende come le Cortes del 1810 si definiscano generales y extraordinarias -e non costituenti- secondo una terminologia di Antico Regime.

Generales in quanto comprendenti i rappresentanti degli antichi regni di Castiglia e di Aragona, come stabilito dai Decretos de Nueva Planta del 1707 e del 1716, che, tra le varie disposizioni, abolivano, per l'appunto, le Cortes aragonesi; e, anche, extraordinarias, dizione solitamente usata per gli affari che travalicavano l'ordinaria amministrazione, come il caso di imposizione di tributi in determinate circostanze (ad esempio per far fronte a impreviste spese belliche).

\footnotetext{
${ }^{39}$ Sul punto si rimanda, tra i molti, a S.M. Coronas González, "El pensamiento constitucional de Jovellanos", Historia constitucional: Revista Electrónica de Historia Constitucional, n. 1, 2000, 63-96 (http://www.historiaconstitucional.com/).

${ }^{40} \mathrm{Per}$ il testo consultato v. http://www.dircost.unito.it/ cit (anche con traduzione in italiano della costituzione del 1812).
} 
Da ultimo, il rispetto delle forme precedenti va ricercato nell'elezione a Cadice dei soli diputados dell' Estado llano (in rappresentanza del territorio metropolitano, ma, questa fu la grande novità, anche dei domini coloniali), dapprima secondo le disposizioni della Suprema Junta Central e in seguito in base alle Istruzioni della Regencia $^{41}$, che sancirono la riunione dei diputados in un'unica Camera ${ }^{42}$.

Decisione quest'ultima certamente sofferta e a lungo dibattuta in seno alla Regencia e nell'opinione pubblica fin dall'inizio della Guerra di Indipendenza e resa nota il 20 settembre 1810, solo quattro giorni prima dell'apertura delle Cortes, momento in cui esse, con un vero e proprio golpe, assunsero la sovranità popolare ${ }^{43}$, anche se occorrerà attendere un mese e mezzo perché le stesse potessero essere definite propriamente "costituenti"; in ciò seguendo l'esortazione del deputato equadoregno, Mejía Lequerica, che con fatidiche parole, chiese, l' 8 dicembre 1810, che l'assemblea non si sciogliesse fino a che non avesse redatto una costituzione politica.

$\mathrm{Fu}$ così nominata una commissione con l'incarico di redigere un progetto di costituzione ${ }^{44}$.

\section{Una breve conclusione}

Le forme dell'Antico Regime risultavano rispettate già con l'art. 1 del Capitolo VI (De la elección de Diputados de las ciudades de voto en Cortes) della Convocatoria para las Juntas superiores (1 gennaio 1810), ad opera della Suprema Junta Central ${ }^{45}$; Juntas che dovranno eleggere le Cortes in rappresentanza della Spagna sollevata contro l'occupante francese.

Todas las ciudades que a las últimas Cortes celebradas en el año de 1789 enviaron Diputados, enviarán uno para éstas.

In qualche modo sembra dunque rivivere il quadro istituzionale dell'Antico Regime, pur in assenza di Ferdinando VII e della stessa Monarchia: in una parola le

\footnotetext{
${ }^{41}$ Anche se non si poteva escludere un'estrazione nobiliare o ecclesiastica dei diputados (infatti gli appartenenti allo stato ecclesiastico rappresentarono la maggioranza dell'assemblea di Cadice), eletti nell'estamento popular, salvo espressi divieti, come nel caso dell'esclusione degli appartenenti ad ordini sacri che seguissero una regola -i c.d. regulares-, anche, in seguito, in ragione del "silenzio" dell'art. 35 della costituzione del 1812 ("Ogni Congresso elettorale di parrocchia si comporrà di tutti i cittadini, domiciliati e residenti nel territorio di essa, compresi gli ecclesiastici secolari.").

${ }^{42}$ Art. 29 ss.; tipo di elezioni, queste su tre gradi --tra cui il primo da tenersi nelle parrocchie- che richiamano alla mente quelle di elezione dei deputati del Terzo Stato in alcune zone rurali in Francia nel 1789.

${ }^{43}$ Acta de instalación de las Cortes generales y extraordinarias (http://bib.cervantesvirtual.com/portal/1812/juntista.shtml).

${ }^{44}$ M. Friera Álvarez e I. Fernández Sarasola, Contexto histórico, e, per i resoconti, Diario de sesiones de las Cortes Generales y Extraordinarias: dieron principio el 24 de setiembre de 1810 y terminaron el 20 de setiembre de 1813, Madrid, Imprenta de J.A. García, 1870-1874, vol. 1, 153.

${ }^{45} \mathrm{http}: / /$ bib.cervantesvirtual.com/portal/1812/juntista.shtml.
} 
Cortes non cancellano la propria eredità politica, anche dopo la riunione del 1789. Infatti non può dirsi che, per quanto concerne la procedura di convocazione e la riunione delle Cortes di Cadice, il passato sia stato dimenticato, giacché, come si è visto, esse non nascono costituenti. E vi è di più: a conclusione di queste poche considerazioni si può pensare al recupero nelle stesse del ruolo dell'elemento popular o ciudadano dell'Estado Llano o General, sopito negli anni del Settecento, ma così rilevante a seguito delle Cortes di Toledo del 1538-39.

Forse, si può aggiungere con cautela, un'influenza assai più decisiva di quella dello stesso modello unicamerale francese del 1791.

Altra questione importante risulta quella della extralimitación dei poteri dei procuradores, come già avvenne nel 1789 in Francia e, ancor prima, nella Convenzione di Filadelfia del 1787, senza dimenticare il momento lato sensu costituente del Parlamento inglese al termine della Gloriosa Rivoluzione (1688-89), riguardante il nuovo patto tra le Camere e il nuovo re, Guglielmo d'Orange.

Così, nelle varie realtà, si arrivò alla riforma dello Stato, della politica e in una parola sola della costituzione senza il carattere cruento delle forme rivoluzionarie; tale tendenza si avrà anche nel pieno Ottocento spagnolo in cui le Cortes, organo legislativo, modificheranno più volte la costituzione scritta, ma sempre nel rispetto della sovranità divisa tra Cortes e Corona.

Lungo tale linea politica si pone anche la stessa costituzione del 1876, quella ispirata dal conservatore Antonio Cánovas del Castillo, più volte Primo Ministro nel corso dell'ultimo quarto del secolo XIX; tale costituzione, ad oggi, è quella che ha goduto della più duratura vigenza nella storia politica spagnola ${ }^{46}$, che si apre nel 1808 con la concessione dell'afrancesado Statuto filo-napoleonico di Baiona ${ }^{47}$.

Tornando infine alle Cortes del 1789, le ultime di Antico Regime, le stesse si discostano certo dall'esperienza gaditana e dalle successive assemblee costituenti della Spagna del secolo XIX.

Tuttavia quelle degli anni 1810-1812 non dimenticano la loro lunga eredità politica; le stesse sono volte ad operare sulle preesistenti leggi fondamentali.

E se il moderantismo ottocentesco governerà, sotto diverse forme, la scena politica spagnola dell'Ottocento, questo sarà anche grazie all'eredità portata avanti fino all'ultimo dalle Cortes del 1789.

Cortes che, nella loro composizione del 1789, in ragione della quale erano convocati i soli rappresentanti dell'Estado llano, appaiono, anche per questo, straordinariamente moderne.

\footnotetext{
${ }^{46}$ Il testo del 1876 venne adottato al termine di una altra esperienza certamente exaltada (e caotica), quella della Prima Repubblica (1873-74). Alla base vi era sempre l'idea anglofila di Jovellanos sulla costituzione storica.

${ }^{47}$ Per un quadro costituzionale a partire dal 1808 si rimanda a E. Attard, El constitucionalismo español, 1808-1978: ensayo histórico-jurídico, Valencia, 1988.
} 